\title{
Centrality and system size dependence of multiplicity fluctuations in nuclear collisions at $158 \mathrm{~A} \mathrm{GeV}$
}

\author{
C. Alt, ${ }^{9}$ T. Anticic, ${ }^{23}$ B. Baatar, ${ }^{8}$ D. Barna,${ }^{4}$ J. Bartke, ${ }^{6}$ L. Betev,${ }^{10}$ H. Białkowska,${ }^{20}$ C. Blume, ${ }^{9}$ B. Boimska,${ }^{20}$ M. Botje ${ }^{1}$
} J. Bracinik, ${ }^{3}$ R. Bramm, ${ }^{9}$ P. Bunčić, ${ }^{10}$ V. Cerny, ${ }^{3}$ P. Christakoglou, ${ }^{2}$ P. Chung,,${ }^{19}$ O. Chvala, ${ }^{14}$ J. G. Cramer, ${ }^{16}$ P. Csató, ${ }^{4}$ P. Dinkelaker, ${ }^{9}$ V. Eckardt, ${ }^{13}$ D. Flierl, ${ }^{9}$ Z. Fodor, ${ }^{4}$ P. Foka, ${ }^{7}$ V. Friese,,${ }^{7}$ J. Ǵl, ${ }^{4}$ M. Gaździcki, ${ }^{9}, 11$ V. Genchev, ${ }^{18}$

G. Georgopoulos, ${ }^{2}$ E. Gładysz, ${ }^{6}$ K. Grebieszkow, ${ }^{22}$ S. Hegyi,${ }^{4}$ C. Höhne, ${ }^{7}$ K. Kadija,${ }^{23}$ A. Karev, ${ }^{13}$ D. Kikola, ${ }^{22}$ M. Kliemant, ${ }^{9}$ S. Kniege, ${ }^{9}$ V. I. Kolesnikov, ${ }^{8}$ E. Kornas, ${ }^{6}$ R. Korus, ${ }^{11}$ M. Kowalski, ${ }^{6}$ I. Kraus,${ }^{7}$ M. Kreps,${ }^{3}$ A. Laszlo, ${ }^{4}$ R. Lacey, ${ }^{19}$ M. van Leeuwen, ${ }^{1}$ P. Lévai, ${ }^{4}$ L. Litov, ${ }^{17}$ B. Lungwitz, ${ }^{9}$ M. Makariev, ${ }^{17}$ A. I. Malakhov,${ }^{8}$ M. Mateev, ${ }^{17}$ G. L. Melkumov, ${ }^{8}$ A. Mischke, ${ }^{1}$ M. Mitrovski, ${ }^{9}$ J. Molnár, ${ }^{4}$ St. Mrówczyński, ${ }^{11}$ V. Nicolic, ${ }^{23}$ G. Pálla, ${ }^{4}$ A. D. Panagiotou, ${ }^{2}$ D. Panayotov, ${ }^{17}$ W. Peryt, ${ }^{22}$ A. Petridis, ${ }^{2, *}$ M. Pikna, ${ }^{3}$ J. Pluta,,${ }^{22}$ D. Prindle, ${ }^{16}$ F. Pühlhofer, ${ }^{12}$ R. Renfordt, ${ }^{9}$ C. Roland,${ }^{5}$ G. Roland,${ }^{5}$ M. Rybczyński, ${ }^{11, \dagger}$ A. Rybicki, ${ }^{6}$ A. Sandoval, ${ }^{7}$ N. Schmitz, ${ }^{13}$ T. Schuster, ${ }^{9}$ P. Seyboth,${ }^{13}$ F. Siklér, ${ }^{4}$ B. Sitar, ${ }^{3}$ E. Skrzypczak,${ }^{21}$ M. Slodkowski, ${ }^{22}$ G. Stefanek, ${ }^{11}$ R. Stock, ${ }^{9}$ C. Strabel, ${ }^{9}$ H. Ströbele, ${ }^{9}$ T. Susa, ${ }^{23}$ I. Szentpétery, ${ }^{4}$ J. Sziklai, ${ }^{4}$ M. Szuba, ${ }^{22}$ P. Szymanski, ${ }^{10,20}$ V. Trubnikov, ${ }^{20}$ D. Varga,,${ }^{4,10}$ M. Vassiliou, ${ }^{2}$ G. I. Veres, ${ }^{4,5}$ G. Vesztergombi, ${ }^{4}$ D. Vranić, ${ }^{7}$ A. Wetzler, ${ }^{9}$ Z. Włodarczyk, ${ }^{11}$

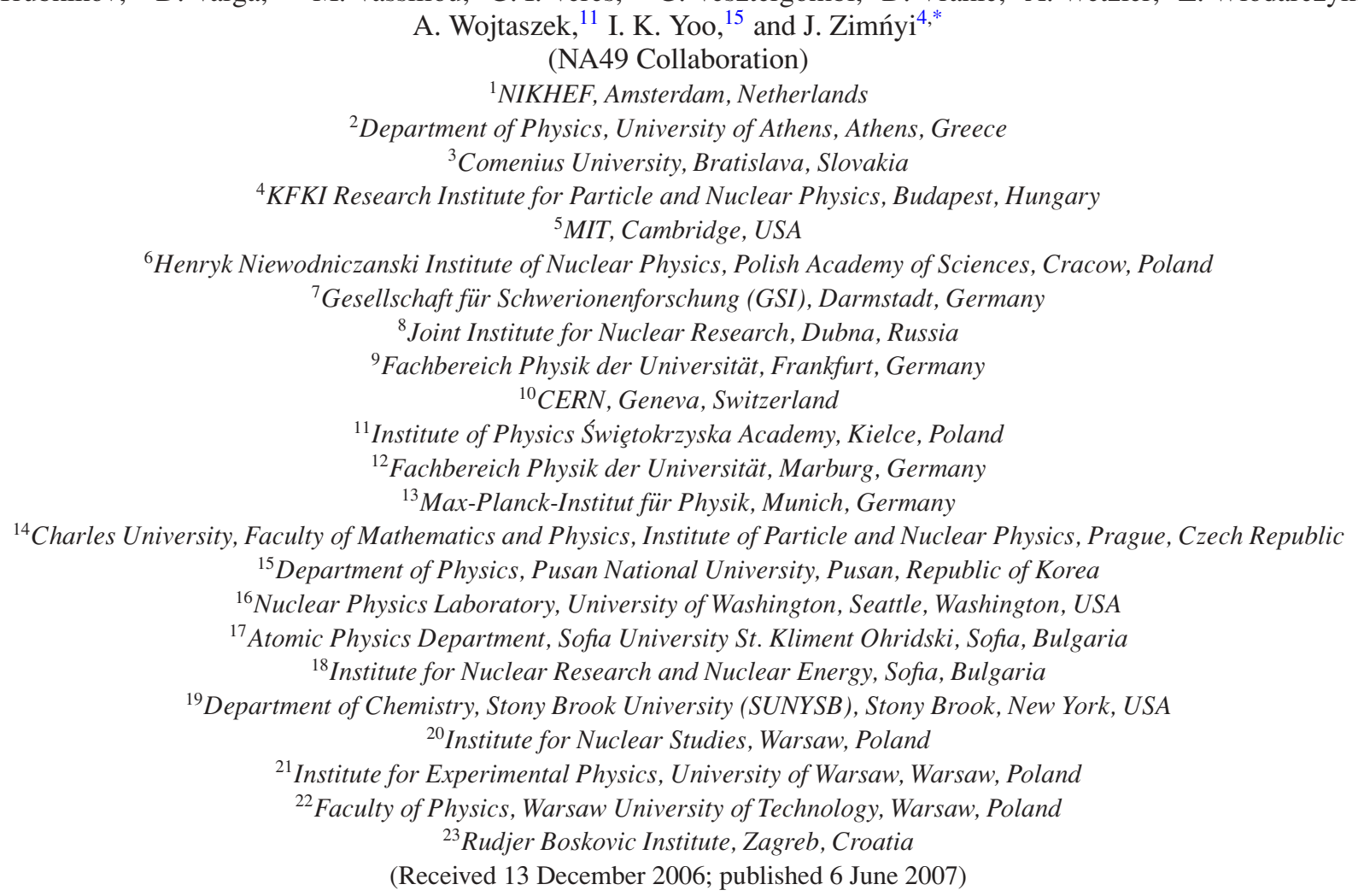

The centrality and system size dependence of multiplicity fluctuations of charged particles produced in nuclear collisions at $158 \mathrm{~A} \mathrm{GeV}$ was studied by the NA49 Collaboration. Centrality selected $\mathrm{Pb}+\mathrm{Pb}$ collisions, semicentral $\mathrm{C}+\mathrm{C}$ and $\mathrm{Si}+\mathrm{Si}$ collisions as well as inelastic $p+p$ interactions were analyzed. The number of projectile participants determined on an event-by-event basis was used to characterize the collision centrality. The scaled variance of the multiplicity distribution obtained in the forward rapidity region $\left(1.1<y_{\text {c.m. }}<2.6\right)$ shows a significant increase toward peripheral collisions. The results are similar for negatively and positively charged particles and about 50\% larger for all charged particles. String-hadronic models of nuclear reactions without the fusion process do not reproduce the rise of fluctuations from central toward peripheral collisions. The measured centrality dependence can be reproduced in superposition models with the assumption of contributions from target participants to particle production in the forward hemisphere or in string models with fusion.

*Deceased.

†mryb@pu.kielce.pl

\section{INTRODUCTION}

Nucleus-nucleus collisions at relativistic energies have been intensely studied over the last two decades. The main goal 
of these efforts is to understand the properties of strongly interacting matter under extreme conditions of high energy and baryon densities for which the creation of a quarkgluon plasma (QGP) is expected [1,2]. Various collision characteristics and their collision energy dependence suggest that a transient state of deconfined matter is created at collision energies as low as $30 \mathrm{~A} \mathrm{GeV} \mathrm{[3].} \mathrm{Fluctuations} \mathrm{of} \mathrm{physical}$ observables in heavy-ion collisions have been a topic of interest for some years as they may provide important signals regarding the formation of a QGP. With the large number of particles produced in heavy-ion collisions at CERN SPS and BNL RHIC energies it has now become feasible to study fluctuations on an event-by-event basis [4]. In a thermodynamical picture of the strongly interacting system formed in the collision, the experimentally studied fluctuations of particle multiplicities [5-9], mean transverse momenta [10-13], and other global observables, are related to fundamental properties of the system, such as specific heat $[14,15]$, chemical potential, and matter compressibility [16]. These, in turn, may reveal information on the properties of the equation of state near the QCD phase boundary $[15,17,18]$.

The main objective of this work is to study how the multiplicity fluctuations change with the varying number of nucleons participating in the collision, with centrality and system size. First results on the centrality dependence in $\mathrm{Pb}+\mathrm{Pb}$ collisions at $158 \mathrm{~A} \mathrm{GeV}$ published by the WA98 Collaboration [5] indicated that the scaled variance of the multiplicity distribution near midrapidity increases toward peripheral collisions. Our analysis extends such measurements to $\mathrm{C}$ and $\mathrm{Si}$ nuclei and carefully addresses the contribution from fluctuations of the number of participants which can dominate the fluctuations of extensive quantities such as the produced particle multiplicity. Furthermore an extensive comparison of the results to available models is performed. The data sample consists of centrality tagged minimum bias $\mathrm{Pb}+\mathrm{Pb}$, semicentral $\mathrm{C}+\mathrm{C}$ and $\mathrm{Si}+\mathrm{Si}$, as well as minimum bias $p+p$ collisions registered by the NA49 detector at the CERN SPS. The multiplicity fluctuations were studied in the forward rapidity region $\left(1.1<y_{\text {c.m. }}<2.6\right)$. The collision centrality was characterized by the number of projectile participants derived from the projectile spectator energy measured in a forward calorimeter. Selection of narrow intervals in this energy minimizes the variation of the number of projectile participants. Although the number of projectile and target participants are closely correlated, the number of target participants cannot be tightly constrained with this procedure. The implications of this problem will be discussed in detail.

The article is organized as follows. In Sec. II the method of measuring multiplicity fluctuations is introduced and discussed. The NA49 setup is presented in Sec. III. Experimental procedures, in particular, event and particle selection, detector acceptance, and centrality determination, are discussed in Sec. IV. The results on centrality and system size dependence of multiplicity fluctuations are presented in Sec. V. In Sec. VI our results are compared with those of other experiments. Possible explanations of our results are discussed in Sec. VII. The article closes with a summary and conclusions.

\section{MULTIPLICITY FLUCTUATIONS}

\section{A. Observables}

Let $P(N)$ be the multiplicity distribution, then

$$
\langle N\rangle=\sum N P(N)
$$

is the mean value of the distribution. The variance of the multiplicity distribution is defined as

$$
\operatorname{Var}(N) \equiv \sum(N-\langle N\rangle)^{2} P(N)=\left\langle N^{2}\right\rangle-\langle N\rangle^{2} .
$$

Note, that for a Poisson distribution the variance equals the mean value, $\operatorname{Var}(N)=\langle N\rangle$. Mean value and variance of multiplicity distributions are the only observables used in this analysis.

\section{B. Participants and spectators}

In a description of nuclear collisions the concept of participant and spectator nucleons is very useful. The participants are nucleons that are removed from the Fermi spheres of target or projectile nuclei due to the collision. The remaining nucleons are called spectators. In the case of central nucleus-nucleus collisions, where the impact parameter $b$ is relatively small, almost all nucleons participate in the collision. In particular, the number of projectile participants, $N_{P}^{\mathrm{PROJ}}$, approximately equals the total number of projectile nucleons $N_{P}^{\mathrm{PROJ}} \approx A$. If the collision is peripheral (with large impact parameter $b$ ) almost all nucleons are spectators, $N_{P}^{\mathrm{PROJ}} \ll A$. The number of projectile spectators $N_{\mathrm{SPEC}}^{\mathrm{PROJ}}$ is given by $N_{\mathrm{SPEC}}^{\mathrm{PROJ}}=A-N_{P}^{\mathrm{PROJ}}$.

\section{Multiplicity fluctuations in superposition models}

Superposition models of nuclear collisions, which are frequently used, assume that secondary particles are emitted by independent sources. A prominent example is the wounded nucleon model [19], in which the sources are wounded nucleons, i.e., the nucleons that have interacted at least once. In this model the number of participants would be equal to the number of wounded nucleons.

In superposition models the total multiplicity is given by

$$
N=\sum_{i=1}^{N_{S}} m_{i},
$$

where $N_{S}$ denotes the number of sources and $m_{i}$ is the multiplicity of secondaries from the $i$-th source. When the sources are identical and independent from each other, the mean total multiplicity equals

$$
\langle N\rangle=\left\langle N_{S}\right\rangle\langle m\rangle,
$$

where $\left\langle N_{S}\right\rangle$ is the mean number of sources and $\langle m\rangle$ is the mean multiplicity from a single source. The variance of the 
multiplicity distribution is

$$
\operatorname{Var}(N)=\left\langle N_{S}\right\rangle \operatorname{Var}(m)+\langle m\rangle^{2} \operatorname{Var}\left(N_{S}\right),
$$

where $\operatorname{Var}(m)$ and $\operatorname{Var}\left(N_{S}\right)$ denote the variance of the distribution of single-source multiplicity and the variance of the distribution of source number, respectively.

The scaled variance of the multiplicity distribution $\operatorname{Var}(N) /\langle N\rangle$ is a useful measure of the multiplicity fluctuations. From Eqs. (4) and (5) one gets

$$
\frac{\operatorname{Var}(N)}{\langle N\rangle}=\frac{\operatorname{Var}(m)}{\langle m\rangle}+\langle m\rangle \frac{\operatorname{Var}\left(N_{S}\right)}{\left\langle N_{S}\right\rangle} .
$$

Thus, in superposition models the measured scaled variance of the multiplicity distribution is the sum of two contributions. The first one describes the multiplicity fluctuations from a single source, whereas the second one accounts for the fluctuations of the number of sources. To infer the fluctuations of main interest given by the first term in Eq. (6) we try to minimize the contribution of the second term. In this experiment events with a fixed number of projectile participants can be selected in which, however, the number of target participants can still fluctuate. The effect of the fluctuation of target participant number at fixed number of projectile participants was theoretically studied in Ref. [20]. Multiplicity fluctuations are measured in a forward rapidity window in which one mainly expects particles produced from projectile participants. Nevertheless the effect of remaining fluctuations of the number of target participants can remain important, if participants produce particles over a wide rapidity range. We return to this point in Secs. IV F and VII.

\section{NA49 EXPERIMENTAL SETUP}

The NA49 experiment is a large acceptance hadron spectrometer at the CERN-SPS (see Fig. 1 and [21]) used to study the final hadronic states produced in collisions of beam particles ( $\mathrm{Pb}$ directly from the SPS, C, and Si via fragmentation of the primary $\mathrm{Pb}$ beam, $p$ as secondary beam from the $450 \mathrm{GeV}$ proton beam in the SPS) with a variety of fixed targets. The main tracking devices are four large-volume time projection chambers (TPCs) that are capable of detecting $60 \%$ of some 1500 charged particles created in a central $\mathrm{Pb}+\mathrm{Pb}$ collision at $158 \mathrm{~A} \mathrm{GeV}$. Two of them, the vertex TPCs (VTPC-1 and VTPC-2), are located inside the magnetic field of two super-conducting dipole magnets (1.5 and 1.1 $\mathrm{T}$, respectively) and two others (MTPC-L and MTPC-R) are positioned downstream of the magnets symmetrically to the beam line. The results presented here are analyzed with a global tracking scheme [22], which combines track segments that belong to the same physical particle but were detected in different TPCs. The NA49 TPCs allow precise measurements of particle momenta $p$ with a resolution of $\sigma(p) / p^{2} \cong(0.3-7)$. $10^{-4}(\mathrm{GeV} / c)^{-1}$. The setup is supplemented by two time-offlight (TOF) detector arrays and a set of calorimeters.

The targets - C $\left(561 \mathrm{mg} / \mathrm{cm}^{2}\right), \operatorname{Si}\left(1170 \mathrm{mg} / \mathrm{cm}^{2}\right)$ disks, and a $\mathrm{Pb}\left(224 \mathrm{mg} / \mathrm{cm}^{2}\right)$ foil for ion collisions and a liquid hydrogen cylinder (length $20.29 \mathrm{~cm}$ ) for elementary interactions-are positioned about $80 \mathrm{~cm}$ upstream from the VTPC-1.

$\mathrm{Pb}$ beam particles are identified by means of their charge as seen by a helium gas Cherenkov counter $\left(\mathrm{S} 2^{\prime}\right)$ in front of the target. The protons were identified by Cherenkov counters farther upstream of the target. The study of $\mathrm{C}+\mathrm{C}$ and $\mathrm{Si}+\mathrm{Si}$ reactions is possible through the generation of a secondary fragmentation beam that is produced by a primary target ( $1 \mathrm{~cm}$ carbon) in the extracted $\mathrm{Pb}$ beam. Setting the beam line momentum accordingly, a large fraction of all $Z / A=1 / 2$ fragments are transported to the NA49 experiment. On-line selection based on a pulse-height measurement in a scintillator beam counter (S2) is used to select particles with $Z=$ $6,7(\mathrm{C}, \mathrm{N})$ and $Z=13,14,15(\mathrm{Al}, \mathrm{Si}, \mathrm{P})$. Off-line clean-up is achieved by using in addition the energy loss measured by beam position detectors (BPD-1/2/3 in Fig. 1). These detectors consist of pairs of proportional chambers and are placed along

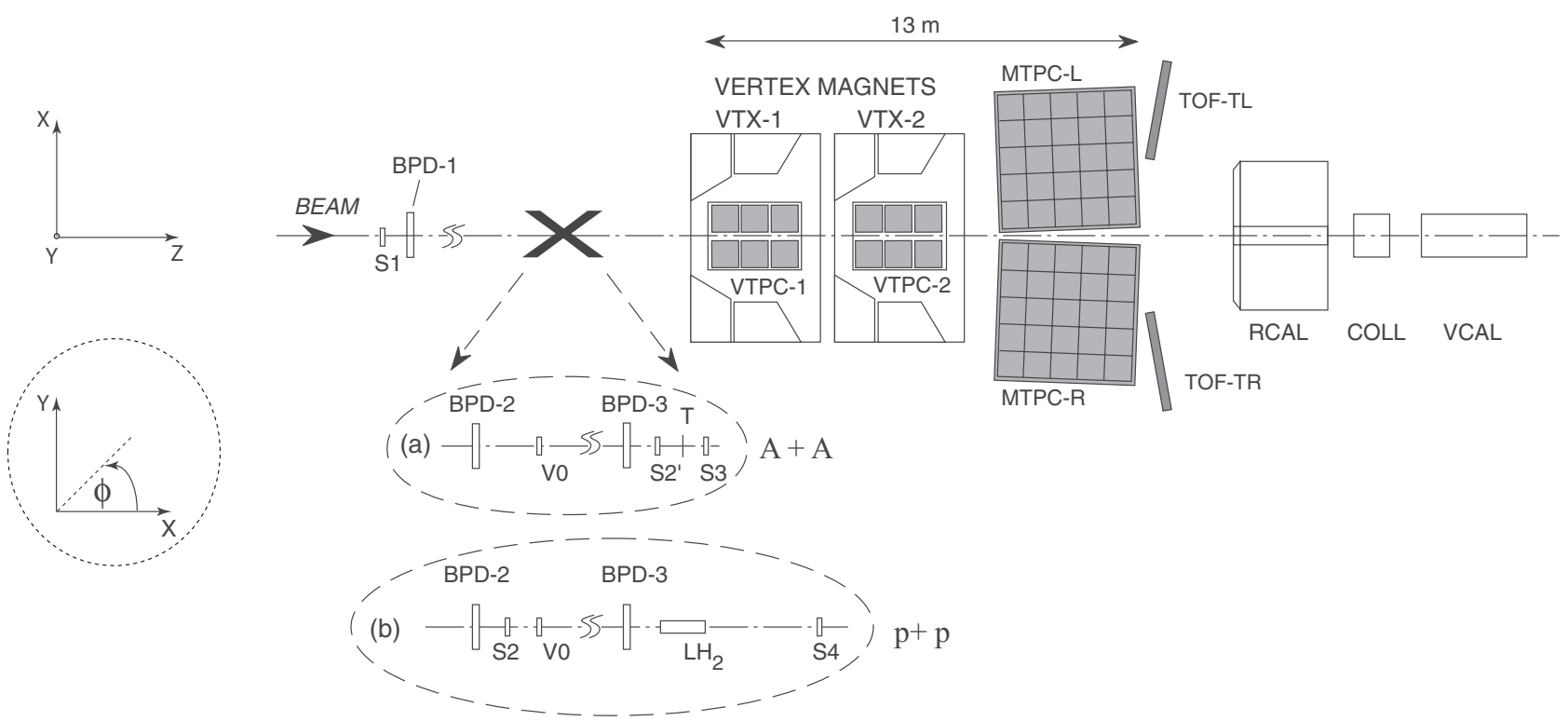

FIG. 1. The experimental setup of the NA49 experiment with different beam definitions and target arrangements. 
the beam line for a precise measurement of the transverse positions of the incoming beam particles.

For $p$ beams, interactions in the target are selected by anticoincidence of the incoming beam particle with a small scintillation counter (S4) placed on the beam trajectory between the two vertex magnets. For $p+p$ interactions at $158 \mathrm{~A} \mathrm{GeV}$ this counter selects a (trigger) cross section of $28.23 \mathrm{mb}$ of $31.78 \mathrm{mb}$ of the total inelastic cross section [23]. For $\mathrm{Pb}$-ion beams an interaction trigger is provided by anticoincidence with a helium gas Cherenkov counter (S3) directly behind the target. The S3 counter is used to select minimum bias collisions by requiring a reduction of the Cherenkov signal by a factor of about 6 . Because the Cherenkov signal is proportional to $Z^{2}$, this requirement ensures that the $\mathrm{Pb}$ projectile has interacted, with a minimal constraint on the type of interaction. This setup limits the triggers on nontarget interactions to rare beam-gas collisions, the fraction of which proved to be small after cuts, even in the case of peripheral $\mathrm{Pb}+\mathrm{Pb}$ collisions.

The centrality of $A+A$ collisions is selected by using information from a veto calorimeter (VCAL), which measures the energy of the projectile spectator nucleons. The geometrical acceptance of the veto calorimeter is adjusted in order to cover the projectile spectator region by a proper setting of the collimator (COLL).

Details of the NA49 detector setup and performance of tracking software are described in Ref. [21].

\section{DATA SELECTION AND ANALYSIS}

\section{A. Data sets}

Multiplicity fluctuations are studied for negatively, positively, and all charged particles selecting events within narrow intervals of energy measured by the VCAL (predominantly energy of projectile spectators). The experimental material used for the analysis consists of samples of $p+p, \mathrm{C}+\mathrm{C}, \mathrm{Si}+\mathrm{Si}$, and $\mathrm{Pb}+\mathrm{Pb}$ collisions at $158 \mathrm{~A} \mathrm{GeV}$. The number of events in each sample is given in Table I. For $\mathrm{Pb}+\mathrm{Pb}$ interactions a minimum bias trigger was used allowing a study of centrality dependence.

\section{B. NA49 acceptance}

The NA49 apparatus detects mainly the particles produced in the forward rapidity hemisphere. Figure 2 shows the

TABLE I. The number of events and the fraction of the total inelastic cross section selected by the on-line trigger for data sets used in this analysis.

\begin{tabular}{crl}
\hline \hline Data set & No. of events & $\sigma / \sigma^{\text {inel }}$ \\
\hline$p+p$ & 319000 & 0.9 \\
$\mathrm{C}+\mathrm{C}$ & 51000 & 0.153 \\
$\mathrm{Si}+\mathrm{Si}$ & 59000 & 0.122 \\
$\mathrm{~Pb}+\mathrm{Pb}$ & 165000 & 0.6 \\
\hline \hline
\end{tabular}

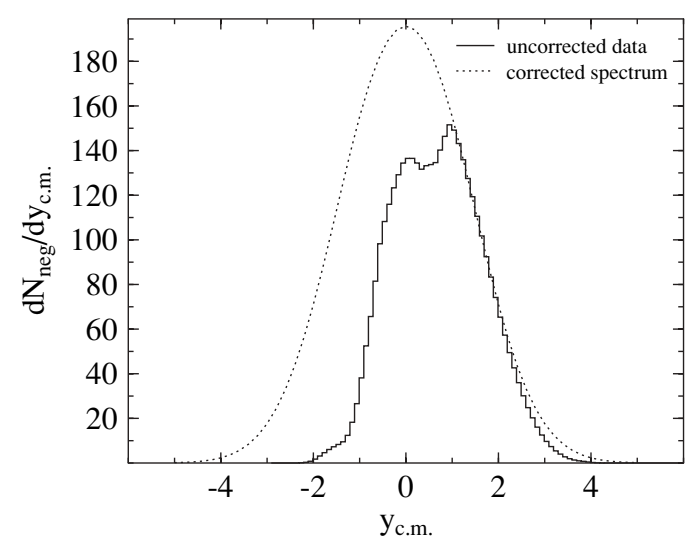

FIG. 2. Rapidity distribution of negatively charged particles produced in central $\mathrm{Pb}+\mathrm{Pb}$ collisions at $158 \mathrm{~A} \mathrm{GeV}$ within the NA49 acceptance (full line) in comparison with an acceptance corrected distribution from Ref. [24] (dotted line).

rapidity distribution of negatively charged particles in the NA49 acceptance calculated assuming that all particles are pions. This distribution is compared to an acceptance corrected rapidity distribution of negatively charged particles from Ref. [24]. As seen, the rapidity region where almost all particles are measured is $1.1<y_{\text {c.m. }}<2.6$.

The NA49 detector was designed for a large acceptance in the forward hemisphere. However, also in this region the geometrical acceptance is not complete. The acceptance limits in the transverse momentum and azimuthal angle were parameterized by a simple function

$$
p_{T}(\phi)=\frac{1}{A+\frac{\phi^{2}}{C}}+B,
$$

where the values of $A, B$, and $C$ depend on the rapidity interval; see Ref. [10]. Only particles within the curves are used in this analysis. This well-defined acceptance is essential for later comparison of the results with models and other experiments. Only forward rapidity tracks $\left(1.1<y_{\mathrm{c} . \mathrm{m} .}<2.6\right.$, rapidity calculated assuming pion mass for all particles) with $0.005<p_{T}<1.5 \mathrm{GeV} / c$ have been used in this analysis.

\section{Event and particle selection}

The aim of the event selection criteria is to reduce contamination from nontarget collisions. The primary vertex was reconstructed by fitting the intersection point of the measured particle trajectories. Only events with a proper quality and position of the reconstructed vertex were accepted for further analysis. The vertex coordinate $Z$ along the beam had to satisfy $\left|Z-Z_{0}\right|<\Delta Z$, where the nominal vertex position $Z_{0}$ and cut parameter $\Delta Z$ values are: -579.5 and $5.5 \mathrm{~cm},-579.5$ and $1.5 \mathrm{~cm},-579.5$ and $0.8 \mathrm{~cm},-581.2$ and $0.6 \mathrm{~cm}$ for $p+p$, $\mathrm{C}+\mathrm{C}, \mathrm{Si}+\mathrm{Si}$, and $\mathrm{Pb}+\mathrm{Pb}$ collisions, respectively. The vertex position in the transverse $X, Y$ coordinates had to agree with the incoming beam position as measured by the BPD detectors. 
To reduce the contamination of particles from secondary interactions, weak decays, and other sources of nonvertex tracks, several track cuts were applied. The accepted particles were required to have measured points in at least one of the vertex TPCs. A cut on the extrapolated distance of closest approach to the fitted vertex of the particle at the vertex plane was applied $\left(\left|d_{X}\right|<4 \mathrm{~cm}\right.$ and $\left.\left|d_{Y}\right|<2 \mathrm{~cm}\right)$. Moreover, the particle was accepted only when the potential number of points (calculated on the basis of the geometry of the track) in the detector exceeded 30 . The ratio of the number of points on a track to the potential number of points had to be higher than 0.5 to avoid split tracks (double counting).

\section{Centrality selection}

To reduce the effect of fluctuations in the number of participants (and thus of particle sources $N_{S}$ ), the multiplicity fluctuations were analyzed in narrow centrality bins defined by the energy measured in the VCAL. This procedure minimizes the variation of the number of projectile participants $N_{P}^{\mathrm{PROJ}}$. Although the number of projectile and target participants are closely correlated, the number of target participants cannot be constrained with the NA49 detector. This leads to a remaining fluctuation in the total number of participants, i.e., particle sources $N_{S}$.

For $\mathrm{C}+\mathrm{C}$ and $\mathrm{Si}+\mathrm{Si}$ interactions, three and five narrow centrality bins were selected, respectively. In the case of $\mathrm{Pb}+\mathrm{Pb}$ collisions, 58 narrow centrality bins were chosen. For each centrality bin the number of projectile participants $N_{P}^{\mathrm{PROJ}}$ was estimated by

$$
N_{P}^{\mathrm{PROJ}}=A-\frac{E_{\mathrm{Veto}}}{E_{\mathrm{LAB}}},
$$

where $E_{\text {Veto }}$ is the energy deposited in the veto calorimeter and $E_{\mathrm{LAB}}$ is the energy carried by single projectile nucleons. The resolution of $E_{\mathrm{Veto}}$, which is recalculated into the resolution of $N_{P}^{\mathrm{PROJ}}$, is discussed in Sec. IV F.

The effect of smearing of energy carried by spectator nucleons due to their Fermi momentum was estimated. The effect was found to be significantly smaller than the resolution of the VCAL and for this reason can be neglected.

We also studied the contribution of nonspectator particles to the energy measured by the veto calorimeter [25] with detailed simulations using VENUS and UrQMD events. The net result was a reduction of the scaled variance of the multiplicity distribution by about 0.3 for central $\mathrm{Pb}+\mathrm{Pb}$ collisions when the nonspectator contribution to the VCAL energy is taken into account. With increasing number of spectators the effect becomes much smaller due to this effect. No corrections were applied to the experimental results, nor were the nonspectator contributions taken into account in the calculations of model predictions.

\section{E. Multiplicity distributions}

The multiplicity distribution depends on the selected $E_{\text {Veto }}$ interval (its position $E_{V}$ and width $\Delta E_{V}$; see Fig. 3 for

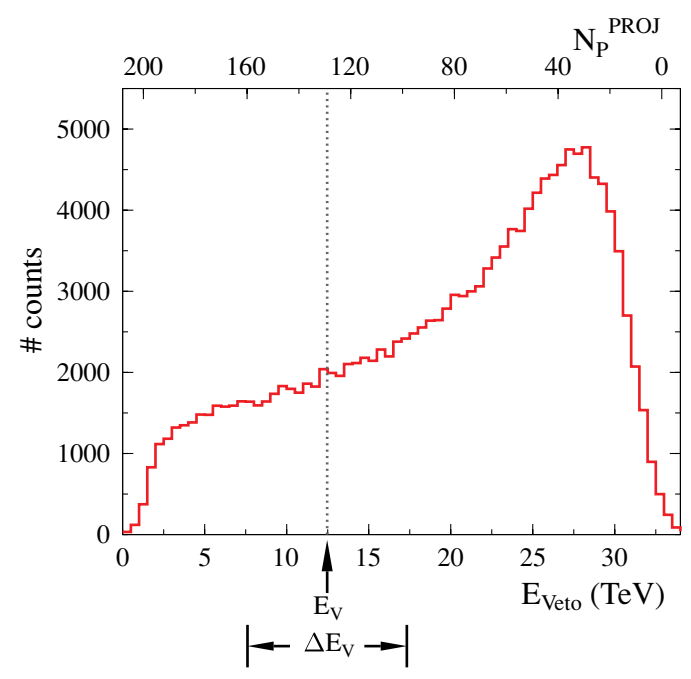

FIG. 3. (Color online) Distribution of energy deposited in the veto calorimeter for minimum bias $\mathrm{Pb}+\mathrm{Pb}$ collisions at $158 \mathrm{~A} \mathrm{GeV}$. An example of an $E_{\text {Veto }}$ interval is shown; the interval is determined by its central value $E_{V}$ and the width $\Delta E_{V}$. The width of the histogram bins corresponds to the width of the $\Delta E_{V}$ intervals used for the fluctuation analysis. The upper horizontal scale shows the corresponding values of the number of projectile participants $N_{P}^{\mathrm{PROJ}}$.

definitions) and the kinematic acceptance selected for the analysis.

In the centrality intervals and acceptance selected for this analysis, multiplicity distributions show approximately Poissonian behavior for $p+p$ and central $\mathrm{Pb}+\mathrm{Pb}$ collisions (see Fig. 4). For semiperipheral collisions the multiplicity distribution is significantly broader than a Poissonian distribution.

In Figs. 5 and 6 the measured mean value and the variance of the multiplicity distributions as a function of the number of projectile participants $N_{P}^{\mathrm{PROJ}}$ are presented. The quantities are not corrected for the fluctuations of the number of projectile participants, as discussed in the next subsection. One can see from these plots that the mean multiplicity shows an approximately linear dependence on the number of projectile participants, whereas the variance of the multiplicity distributions calculated from the data exceeds the variance of the Poisson distributions.

\section{F. Scaled variance of the multiplicity distribution}

The scaled variance of the multiplicity distribution depends on, among other variables, the width of the energy interval $\Delta E_{V}$ selected in the VCAL. For very broad $\Delta E_{V}$ intervals the measured scaled variance of the multiplicity distribution has a large value because of significant fluctuations in the number of projectile participants $N_{P}^{\mathrm{PROJ}}$. Narrowing the $\Delta E_{V}$ interval results in decreasing fluctuations in the number of projectile participants and consequently in a reduction of the scaled variance as shown in Fig. 7. For $\Delta E_{V}$ smaller than about $1 \mathrm{TeV}$ the measured scaled variance of the multiplicity distribution is almost independent of $\Delta E_{V}$. Note that even for very small values of $\Delta E_{V}$ the number of projectile spectators fluctuates due to the finite resolution of the VCAL. In the following 


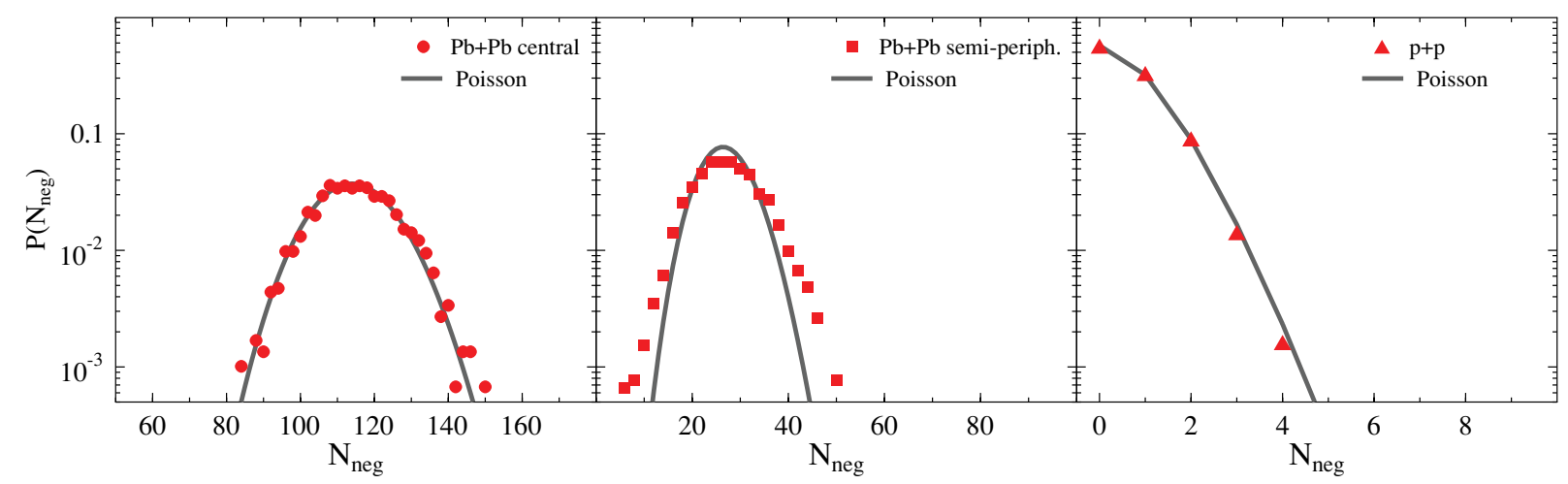

FIG. 4. (Color online) Multiplicity distributions of negatively charged particles obtained for $\Delta E_{V}=500 \mathrm{GeV}$ for central $\mathrm{Pb}+\mathrm{Pb}$ collisions with number of projectile participants $N_{P}^{\mathrm{PROJ}}=178$ (left panel); semiperipheral $\mathrm{Pb}+\mathrm{Pb}$ collisions with $N_{P}^{\mathrm{PROJ}}=39$ (midpanel) and $p+p$ interactions with $N_{P}^{\mathrm{PROJ}}=1$ (right panel).

the scaled variance will be calculated in the $E_{\text {Veto }}$ intervals $\Delta E_{V}=500 \mathrm{GeV}$ (the choice of the width of the interval is a compromise between minimizing correction for the finite interval width and sufficient statistics) and then corrected for fluctuations in the number of projectile participants due to the finite width of the $E_{\text {Veto }}$ interval and the finite resolution of the veto calorimeter.

Within the superposition model leading to Eq. (6), the correction $\delta$ to the scaled variance, which takes into account the finite value of $\Delta E_{V}$ and the calorimeter resolution, is calculated as

$$
\delta=\frac{\langle N\rangle\left[\operatorname{Var}_{\Delta}\left(E_{\text {Veto }}\right)+\operatorname{Var}_{R}\left(E_{\text {Veto }}\right)\right]}{\left(E_{\mathrm{BEAM}}-\left\langle E_{\mathrm{Veto}}\right\rangle\right)^{2}},
$$

where $\operatorname{Var}_{\Delta}\left(E_{\text {Veto }}\right)$ is the variance of $E_{\text {Veto }}$ due to the finite width of the $E_{\text {Veto }}$ bin, $\operatorname{Var}_{R}\left(E_{\text {Veto }}\right)$ is the variance of $E_{\text {Veto }}$ due to the VCAL resolution, $\left\langle E_{\mathrm{Veto}}\right\rangle$ is the mean value of $E_{\text {Veto }}$ in the bin and $E_{\mathrm{BEAM}}=158 \mathrm{~A} \mathrm{GeV}$ is the total beam energy. $\operatorname{Var}_{\Delta}\left(E_{\text {Veto }}\right)$ was calculated from the distribution of $E_{\text {Veto }}$ energy in a given interval. $\operatorname{Var}_{R}\left(E_{\text {Veto }}\right)=\sigma^{2}\left(E_{\text {Veto }}\right)$ with $E_{\text {Veto }}$ expressed in $\mathrm{GeV}$ was parametrized using calibration

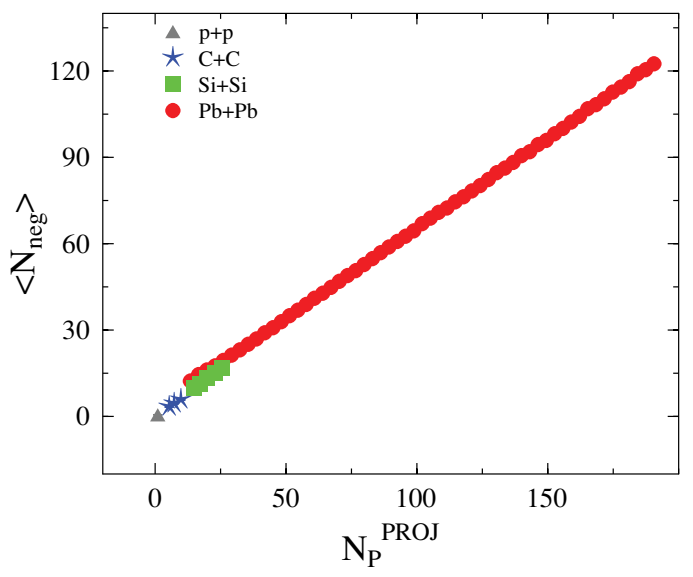

FIG. 5. (Color online) The uncorrected mean value of the multiplicity distribution for negatively charged particles as a function of the number of projectile participants. (rapidity interval $1.1<y_{\text {c.m. }}<2.6$ ) measurements [26] as:

$$
\frac{\sigma\left(E_{\mathrm{Veto}}\right)}{E_{\text {Veto }}}=\frac{2.85}{\sqrt{E_{\text {Veto }}}}+\frac{16}{E_{\text {Veto }}} .
$$

The resolution of $E_{\text {Veto }}$ is easily propagated to the resolution of $N_{P}^{\mathrm{PROJ}}$ using Eq. (8).

Finally, the corrected scaled variance is calculated as

$$
\frac{\operatorname{Var}(n)}{\langle n\rangle}=\frac{\operatorname{Var}(N)}{\langle N\rangle}-\delta,
$$

where $\operatorname{Var}(N)$ is the measured variance, $\langle N\rangle$ the measured mean value of the multiplicity distribution in a given $E_{\text {Veto }}$ bin, and $\delta$ represents the corrections for fluctuations in the number of projectile participants. The dependence of $\delta$ on $\Delta E_{V}$ for some values of $E_{V}$ are presented in Fig. 8, whereas the total correction $\delta$ as a function of number of projectile participants $N_{P}^{\mathrm{PROJ}}$ is presented in Fig. 9. Using the same procedure, corresponding corrections were determined for $\mathrm{C}+\mathrm{C}$ and $\mathrm{Si}+\mathrm{Si}$ collisions. The corrected values of the scaled variance from Eq. (11) are plotted in Fig. 11.

Let us discuss in more detail the physical meaning of the scaled variance displayed in Fig. 11, which is the main result

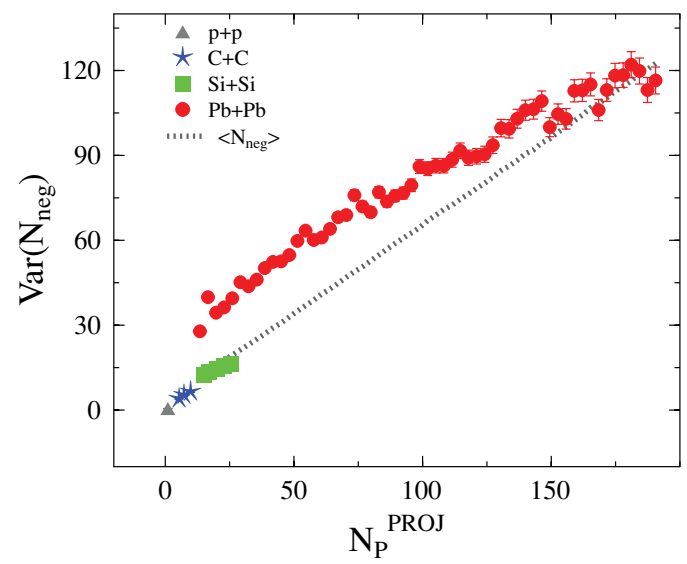

FIG. 6. (Color online) The uncorrected variance of the multiplicity distribution for negatively charged particles as a function of the number of projectile participants. 


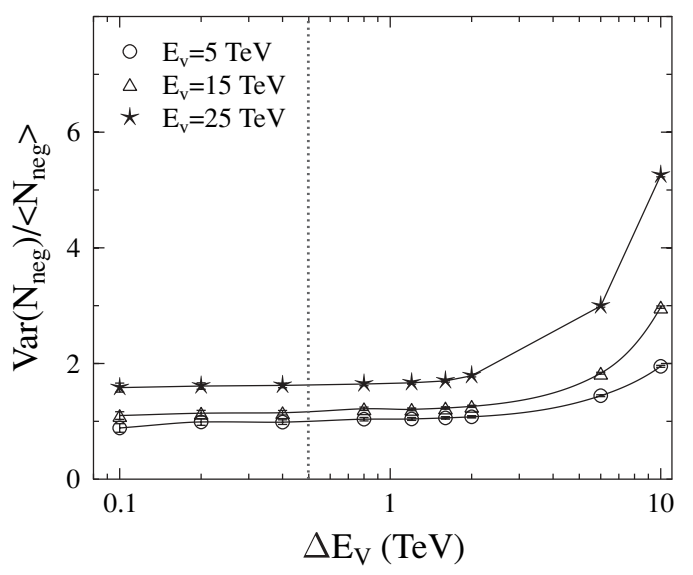

FIG. 7. Measured scaled variance of multiplicity distributions of negatively charged particles for minimum bias $\mathrm{Pb}+\mathrm{Pb}$ collisions as a function of the interval width $\Delta E_{V}$ of the selected VCAL energy for various positions $E_{V}$ of the interval. The vertical line shows the width of the interval, which was used for further analysis. See text for details.

of our study. As already stressed, fluctuations of the number of projectile participants contribute to the multiplicity fluctuations of interest. However, the scaled variance of the multiplicity distribution can be split within the superposition model according to Eq. (6), that is, into the contributions coming from a single participant (or equivalently from a fixed number of participants) and from the varying number of participants- the first and second terms in Eq. (6), respectively. Because the correction $\delta$ represents the contribution from the fluctuating number of projectile participants, the corrected scaled variance shown in Fig. 11 describes fluctuations at fixed number of projectile participants.

If the number of projectile participants uniquely determines the number of particle sources, which contribute to the rapidity interval under study, the scaled variance from Fig. 11

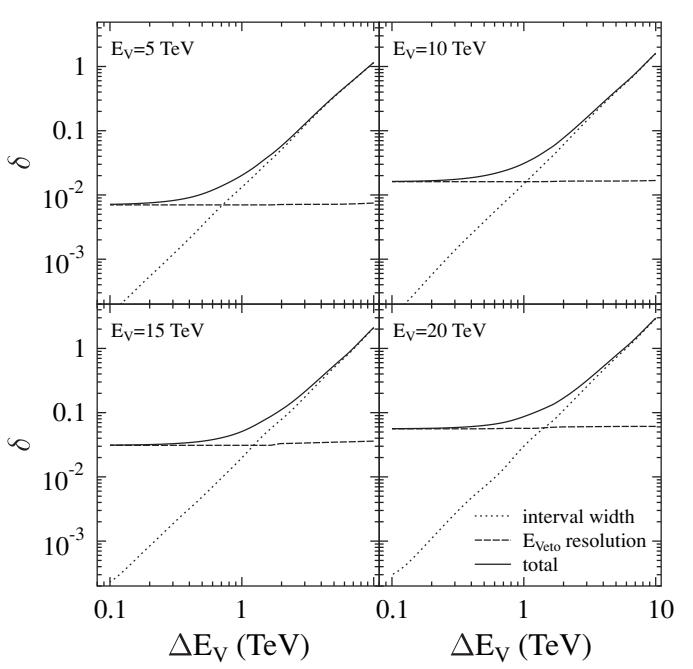

FIG. 8. The corrections for veto calorimeter resolution and finite interval width as a function of interval width $\Delta E_{V}$ for various positions $E_{V}$ of the interval.

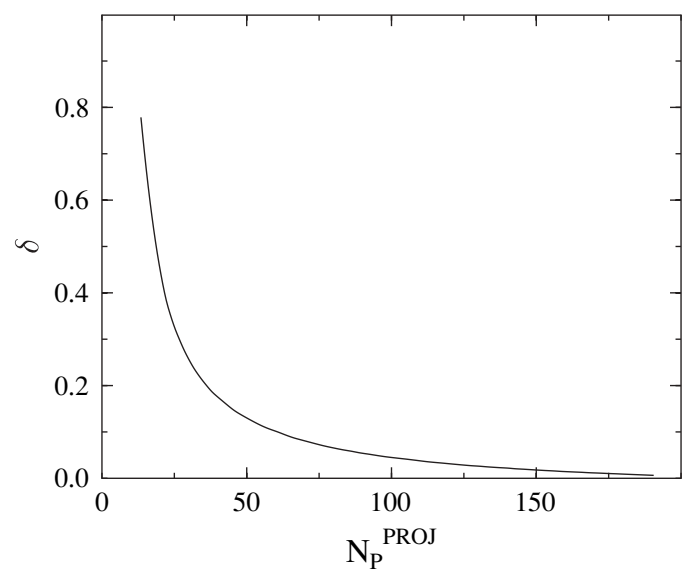

FIG. 9. The total correction applied to the measured scaled variance of negatively charged particles as a function of the number of projectile participants $N_{P}^{\mathrm{PROJ}}$.

represents the multiplicity fluctuations of particles coming from a single source. However, the number of particle sources can fluctuate even at fixed number of projectile participants. For example, the number of strings, which decay into particles, is a random variable in some models. The number of particle sources can also fluctuate due to the fluctuating number of target participants which are not observed in the NA49 detector. Thus, we stress that the scaled variance shown in Fig. 11 represents the multiplicity fluctuations at fixed number of projectile participants and may still contain some contribution from fluctuations in the number of particle sources.

\section{G. Statistical errors}

In general, the variance of a quantity expressed by the fraction $\omega=x / y$ is given as

$$
\operatorname{Var}(\omega) \approx\left(\frac{\langle x\rangle}{\langle y\rangle}\right)^{2}\left[\frac{\sigma_{x}^{2}}{\langle x\rangle^{2}}+\frac{\sigma_{y}^{2}}{\langle y\rangle^{2}}-\frac{2 \operatorname{Cov}(x, y)}{\langle x\rangle\langle y\rangle}\right],
$$

where the symbol $\langle\ldots\rangle$ means an average over events, $\sigma$ denotes the statistical error of $x$ or $y$, and $\operatorname{Cov}(x, y)$ is the covariance between $x$ and $y$. The statistical error of the quantity $\omega$ is given by $\sigma(\omega)=\sqrt{\operatorname{Var}(x / y)}$.

For the calculation of the statistical errors of the scaled variance, we have $x=\operatorname{Var}(N), y=\langle N\rangle$ and

$$
\begin{aligned}
\sigma_{x} & =\frac{\operatorname{Var}(N)}{\sqrt{L}} \sqrt{2+\gamma_{2}}, \quad \sigma_{y}=\frac{\sqrt{\operatorname{Var}(N)}}{\sqrt{L}}, \\
\operatorname{Cov}(x, y) & =\frac{\mu_{3}(N)}{L},
\end{aligned}
$$

where $\gamma_{2}=\mu_{4}(N) / \mu_{2}^{2}(N)-3$ is the kurtosis, $\mu_{k}(N)$ is the $k$-th central moment of the multiplicity distribution, and $L$ is the number of events.

The statistical errors of our results are usually much smaller than the systematic uncertainties discussed in Sec. IV I. 


\section{H. Stability for event cuts and track selection}

In this section the stability of our results with respect to variations of the event and track selection criteria is described. All tests were performed for negatively charged particles produced in $\mathrm{Pb}+\mathrm{Pb}$ minimum bias collisions for the centrality bin corresponding to the number of projectile participants $N_{P}^{\mathrm{PROJ}}=39$.

The event and track selection criteria are designed to reduce the contamination from the background. To check the stability of the results obtained for $\operatorname{Var}\left(n_{\text {neg }}\right) /\left\langle n_{\text {neg }}\right\rangle$ the cut parameters were varied within reasonable ranges. In addition, results obtained from the analysis of data taken at two different magnetic field polarities as well as during different running periods have been compared.

Event cuts are used to reject contamination by nontarget interactions. Figure 10(a) shows the scaled variance as a function of the maximally accepted distance $\Delta Z$ between fitted and nominal $Z$ position of the primary vertex. As one can see, the scaled variance of the multiplicity distribution is stable with respect to a small contamination by nonvertex tracks.

Figure 10(b) shows the scaled variance as a function of the maximally allowed difference of the $X$ and $Y$ position of the fitted main vertex from the transverse beam position given by the beam position detectors (cf. Fig. 1). Also in this case the scaled variance is stable.

The majority of tracks selected by the track selection criteria are main vertex tracks and the remaining fraction $(\approx 10 \%)$ originates predominantly from weak decays and secondary interactions with the material of the detector. To estimate the influence of this contamination on the multiplicity fluctuations, the maximally accepted distance between the extrapolated track and the reconstructed primary vertex in the target plane was varied (see Fig. 10(c)).

Losses of tracks due to reconstruction inefficiency and track selection cuts influence the measured multiplicity fluctuations. To estimate this effect, the dependence of $\operatorname{Var}\left(n_{\text {neg }}\right) /\left\langle n_{\text {neg }}\right\rangle$ on the ratio of the number of points on a track to the potential number of points was determined (see Fig. 10(d)).

In summary, the values of the scaled variance appear stable with respect to reasonable variations in the event and track selection cuts.

\section{Systematic uncertainties}

There are several sources of systematic uncertainties of our results. The systematic error due to the contamination of nonvertex interactions, tracks from weak decays and secondary interactions as well as reconstruction inefficiencies and biases were estimated by varying event and track selection cuts and simulations as discussed in the previous section. The systematic error due to the above mentioned effects was estimated to be $10 \%$.

The main source of a possible systematic bias, however, is the uncertainty in the measurement of the energy deposited in VCAL. To estimate the influence of this uncertainty on the scaled variance of the multiplicity distribution the analysis of so-called beam-only events was performed. Here, the $\mathrm{Pb}$ beam hits into VCAL without interactions with target nuclei. The
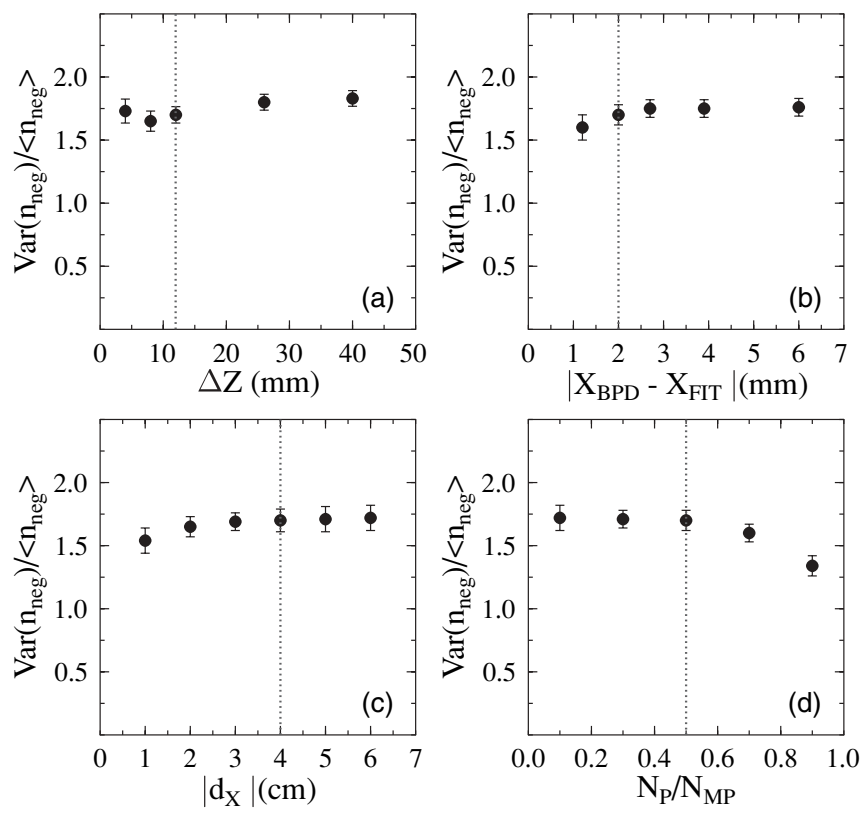

FIG. 10. Scaled variance of the multiplicity distribution of negatively charged particles for the centrality bin corresponding to the number of projectile participants $N_{P}^{\mathrm{PROJ}}=39$ as a function of (a) maximally allowed difference $\Delta Z$ between fitted main vertex and target position; (b) maximally allowed difference between the transverse beam position measured by the BPD and the fitted main vertex position $\left(\left|X_{\mathrm{BPD}}-X_{\mathrm{FIT}}\right|\right)$ (simultaneously the difference in $\left|Y_{\mathrm{BPD}}-Y_{\mathrm{FIT}}\right|$ was required to be below $2 / 3$ of the upper limit in $\left|X_{\mathrm{BPD}}-X_{\mathrm{FIT}}\right|$ ); (c) maximally allowed distance between the reconstructed primary vertex and the track in the target plane $\left|d_{X}\right|$ (simultaneously the deviation in $\left|d_{Y}\right|$ was required to be below $\left.0.5 \cdot\left|d_{X}\right|\right)$; (d) minimum value of the ratio of the number of points on the track $N_{P}$ to the potential number of points $N_{\mathrm{MP}}$. The vertical lines indicate the cuts used to obtain the results.

measured resolution was $\sigma\left(E_{\text {Veto }}\right) / E_{\text {Veto }}=2.5 / \sqrt{E_{\text {Veto }}}\left(E_{\text {Veto }}\right.$ in $\mathrm{GeV}$ ) [26]. However, the beam impinges on a small spot in the VCAL, and the resolution estimated by using beam only events does not take into account the VCAL nonuniformity [27]. To estimate it, the correlation between signals deposited in different sectors of the VCAL were studied. From the result the resolution (see Eq. (10)) of the veto calorimeter was derived [26]. The systematic error due to the finite resolution and nonuniformity of the VCAL was estimated to be smaller than $10 \%$ for $N_{P}^{\mathrm{PROJ}}>20$ and $25 \%$ for $N_{P}^{\mathrm{PROJ}}<20$.

Finally, the systematic error of the results in $\mathrm{Pb}+\mathrm{Pb}$ collisions was set to be equal to $10 \%$ for $N_{P}^{\mathrm{PROJ}}>20$ and $25 \%$ for $N_{P}^{\mathrm{PROJ}}<20$. The resulting estimates are shown by the outer error bars in Fig. 11. For semicentral $\mathrm{C}+\mathrm{C}$ and $\mathrm{Si}+\mathrm{Si}$ collisions we assume 10\% systematic error (cf. Figs. 12-13). In $p+p$ interactions the centrality selection does not apply and the systematic error was estimated by varying event and track selection cuts yielding $10 \%$ error.

\section{RESULTS}

The results discussed in this section refer to the accepted particles, i.e., particles that are registered by the detector and 


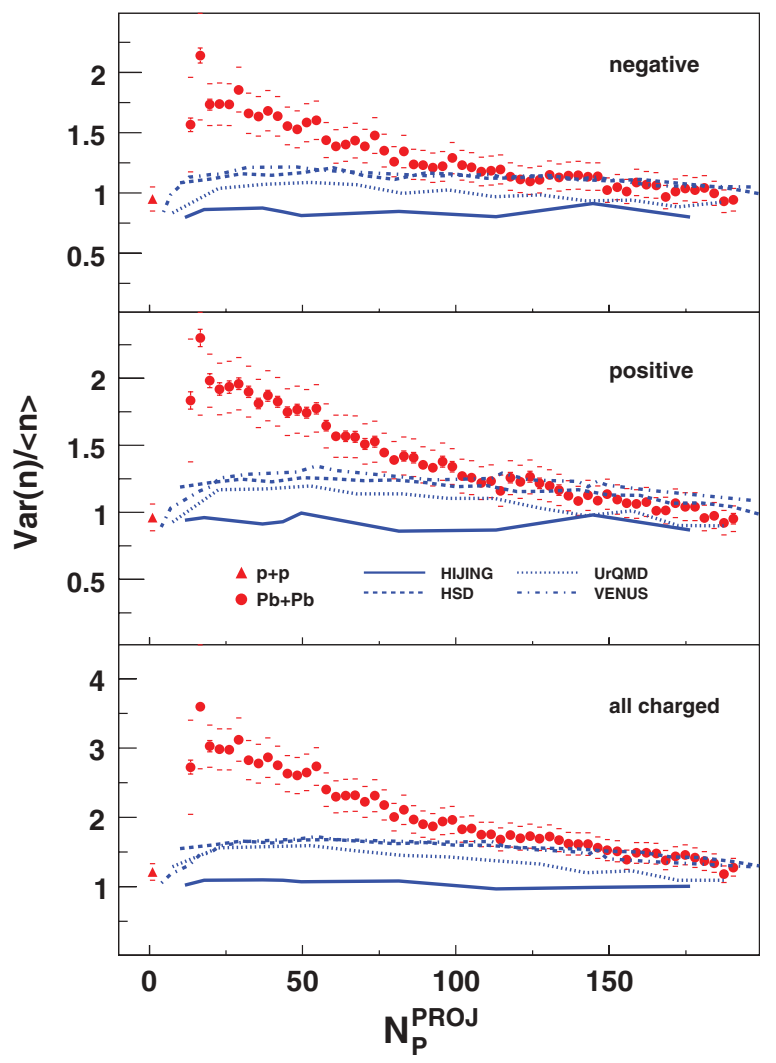

FIG. 11. (Color online) The scaled variance of the multiplicity distribution for negatively (upper panel), positively (middle panel), and all (bottom panel) charged particles as a function of the number of projectile participants $N_{P}^{\mathrm{PROJ}}$ compared with model simulations in the NA49 acceptance (HSD and UrQMD predictions were taken from Ref. [35]). The statistical errors are smaller than the symbols (except for the most peripheral points). The horizontal bars indicate the systematic uncertainties (the upper one for the second point is off scale).

pass all kinematic cuts and track selection criteria. The data cover a broad range in $p_{T}\left(0.005<p_{T}<1.5 \mathrm{GeV} / c\right)$. The center-of-mass rapidity of accepted particles is restricted to the interval $1.1<y_{\text {c.m. }}<2.6$, where the azimuthal acceptance given by Eq. (7) is large.

\section{A. Multiplicity fluctuations in $\mathbf{P b}+\mathbf{P b}$ collisions}

The corrected scaled variance of the multiplicity distribution for negatively, positively, and all charged accepted particles produced in minimum bias $\mathrm{Pb}+\mathrm{Pb}$ collisions as a function of centrality is shown in Fig. 11 (cf. $[3,28]$ ) and compared to HIJING [29], HSD [30], UrQMD [31], and VENUS [32] simulations. In the model calculations a realistic simulation of the determination of the number of projectile participants was included. The models produce approximately Poissonian multiplicity distributions independent of centrality. The data points, in contrast, indicate a strong increase toward peripheral collisions possibly with a maximum at about $N_{P}^{\mathrm{PROJ}} \approx 30$ projectile participants. We note that by chance the scaled variance is approximately 1 for $p+p$ collisions

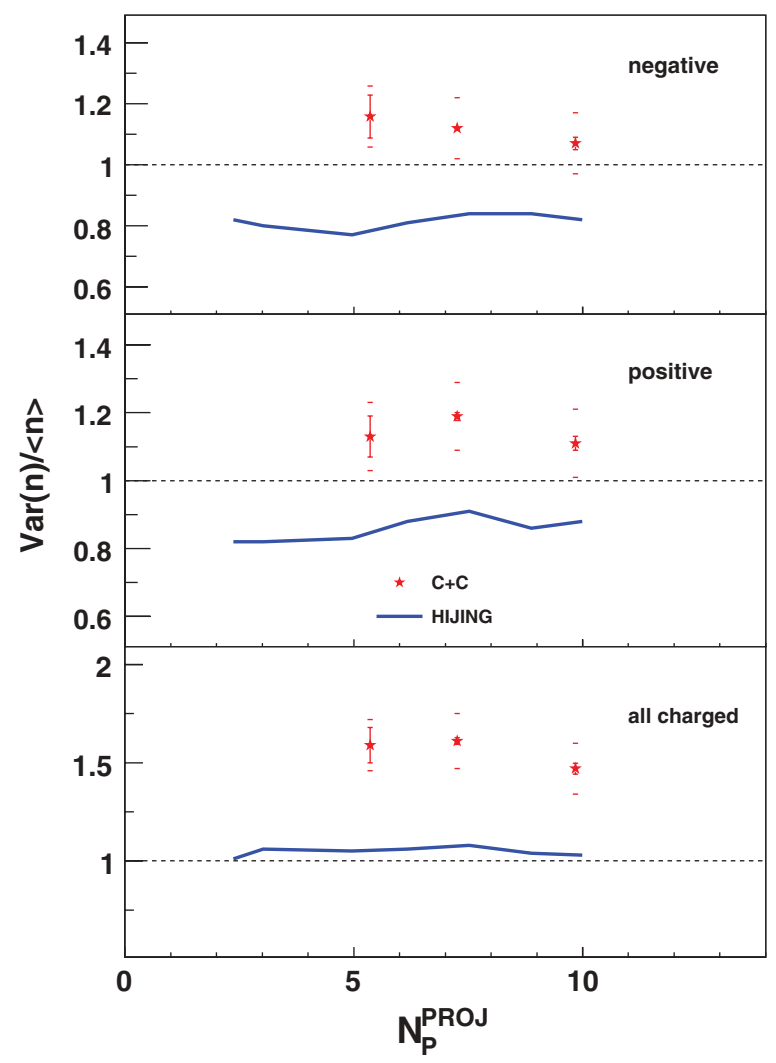

FIG. 12. (Color online) The scaled variance of the multiplicity distribution of negatively (upper panel), positively (middle panel), and all (bottom panel) charged particles produced in semicentral $\mathrm{C}+\mathrm{C}$ collisions as a function of the number of projectile participants $N_{P}^{\mathrm{PROJ}}$ compared with HIJING simulations in the NA49 acceptance. The horizontal bars show the systematic uncertainties, whereas the statistical errors are represented by the vertical bars. Except for the most peripheral points the statistical errors are smaller than the symbols.

at $158 \mathrm{~A} \mathrm{GeV}$, although the multiplicity distribution in $p+p$ collisions is not Poissonian both at lower and higher energies (cf. Sec. VI and Ref. [33]).

The scaled variances for positively and negatively charged particles are similar. The corresponding values for all charged particles are larger. Assuming that negatively and positively charged particles in the experimental acceptance are correlated with a correlation factor $\rho$, one gets

$$
\frac{\operatorname{Var}\left(n_{\mathrm{ch}}\right)}{\left\langle n_{\mathrm{ch}}\right\rangle}=\frac{\operatorname{Var}\left(n_{\mathrm{neg}}\right)}{\left\langle n_{\mathrm{neg}}\right\rangle}(1+\rho)=\frac{\operatorname{Var}\left(n_{\mathrm{pos}}\right)}{\left\langle n_{\mathrm{pos}}\right\rangle}(1+\rho),
$$

where $n_{\text {neg }}, n_{\text {pos }}$, and $n_{\text {ch }}$ are multiplicities of negatively, positively, and all charged particles, respectively, and, for simplicity, we assumed $\left\langle n_{\text {neg }}\right\rangle=\left\langle n_{\text {pos }}\right\rangle$. As seen, the scaled variance for charged particles is $1+\rho$ times as large as that for positives or negatives (see also Ref. [34]). The positive value of the correlation factor required by the data $(\rho \approx 0.5)$ may reflect the effect of electric charge conservation. 


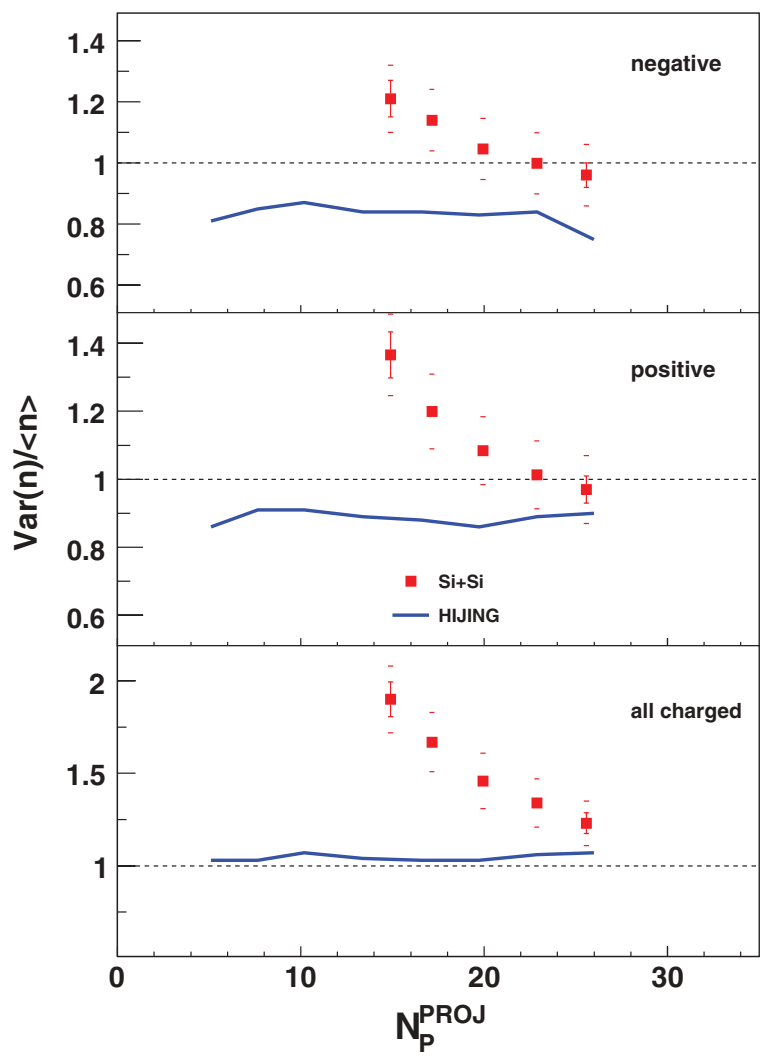

FIG. 13. (Color online) The scaled variance of the multiplicity distribution of negatively (upper panel), positively (middle panel), and all (bottom panel) charged particles produced in semicentral $\mathrm{Si}+\mathrm{Si}$ collisions as a function of the number of projectile participants $N_{P}^{\text {PROJ }}$ compared with HIJING simulation in the NA49 acceptance. The horizontal bars show the systematic uncertainties, whereas the statistical errors are represented by the vertical bars. Except for the most peripheral points the statistical errors are smaller than the symbols.

\section{B. Multiplicity fluctuations in $\mathrm{C}+\mathrm{C}$ and $\mathrm{Si}+\mathrm{Si}$ collisions}

The corrected scaled variances of multiplicity distributions for negatively, positively, and all charged accepted particles produced in semicentral $\mathrm{C}+\mathrm{C}$ and $\mathrm{Si}+\mathrm{Si}$ collisions as a function of centrality are presented in Figs. 12 and 13. The data are compared with results from a HIJING simulation. The scaled variance for negative and positive hadrons shows almost Poissonian fluctuations in $\mathrm{C}+\mathrm{C}$ as well as in $\mathrm{Si}+\mathrm{Si}$ collisions, similarly to the observed multiplicity fluctuations in central $\mathrm{Pb}+\mathrm{Pb}$ interactions. The multiplicity fluctuations for all charged accepted particles are much larger than for the like sign, as also observed in $\mathrm{Pb}+\mathrm{Pb}$ collisions. Because data on peripheral $\mathrm{C}+\mathrm{C}$ and $\mathrm{Si}+\mathrm{Si}$ collisions are not available, it is difficult to speculate about the centrality dependence of the scaled variance. However, it seems that there is a scaling seen in the variable $f=N_{P}^{\mathrm{PROJ}} / A$, the fraction of nucleons participating in the collision (Fig. 14). To validate this very suggestive scaling data on minimum bias collisions of $\mathrm{C}+\mathrm{C}$ and $\mathrm{Si}+\mathrm{Si}$ would be necessary.

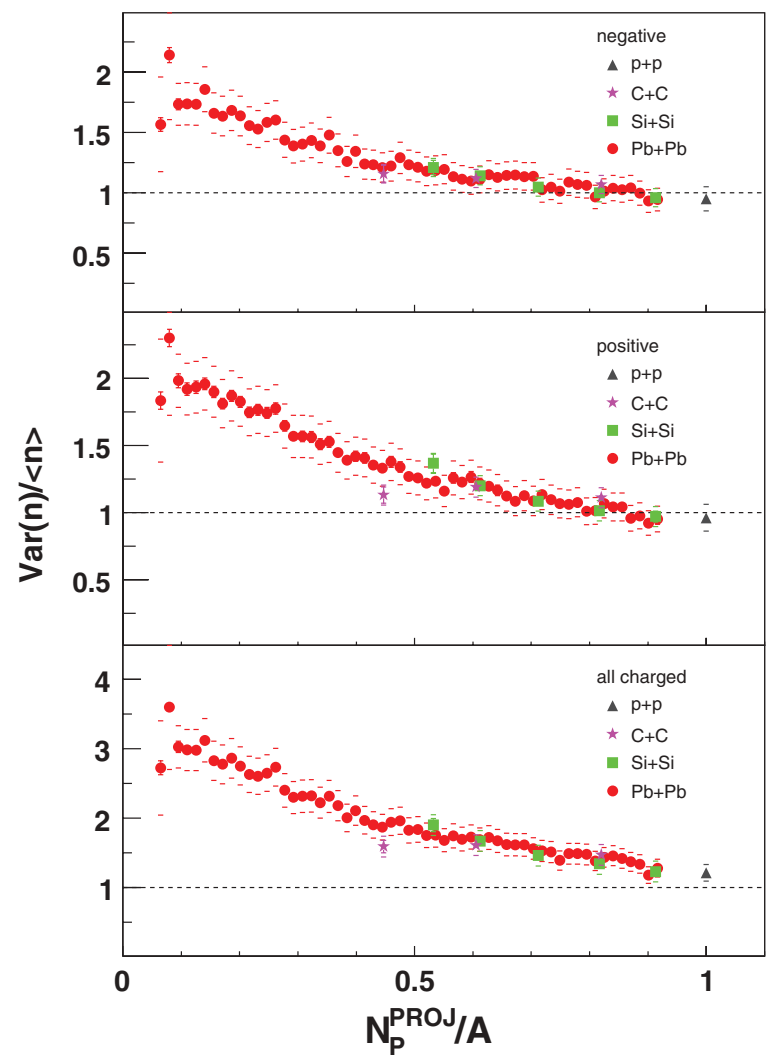

FIG. 14. (Color online) The scaled variance of the multiplicity distribution of negatively (upper panel), positively (middle panel), and all (bottom panel) charged particles produced in $p+p$, semicentral $\mathrm{C}+\mathrm{C}$, semicentral $\mathrm{Si}+\mathrm{Si}$, and $\mathrm{Pb}+\mathrm{Pb}$ collisions as a function of the fraction of nucleons that participate in the collision. The statistical errors are smaller than the symbols (except for the most peripheral points). The horizontal bars indicate the systematic uncertainties (the upper one for the second point is off scale).

\section{COMPARISON WITH RESULTS FROM OTHER EXPERIMENTS}

Assuming that the produced particles are emitted independently in momentum space, the mean multiplicity in a limited acceptance $\langle N\rangle$ can be expressed through the mean multiplicity in the full acceptance $\left\langle N_{p=1}\right\rangle$ as $\langle N\rangle=p\left\langle N_{p=1}\right\rangle$, where $p$ is the fraction of registered particles. The variance of the multiplicity distribution in a limited acceptance can be written as $\operatorname{Var}(N)=p^{2} \operatorname{Var}\left(N_{p=1}\right)+\left\langle N_{p=1}\right\rangle p(1-p)$. The probability that a given fraction of produced particles is registered is given by the binomial distribution. Using the above formulas, one easily finds the scaled variance in a limited acceptance

$$
\frac{\operatorname{Var}(N)}{\langle N\rangle}=1+p\left[\frac{\operatorname{Var}\left(N_{p=1}\right)}{\left\langle N_{p=1}\right\rangle}-1\right],
$$

which depends linearly on the fraction $p$ of registered particles.

To compare to results obtained by other experiments, the NA49 results were extrapolated to the full azimuthal acceptance in the rapidity interval $1.1<y_{\text {c.m. }}<2.6$. The extrapolation was done by means of Eq. (15) and values of $p$ obtained from a HIJING simulation that included the 


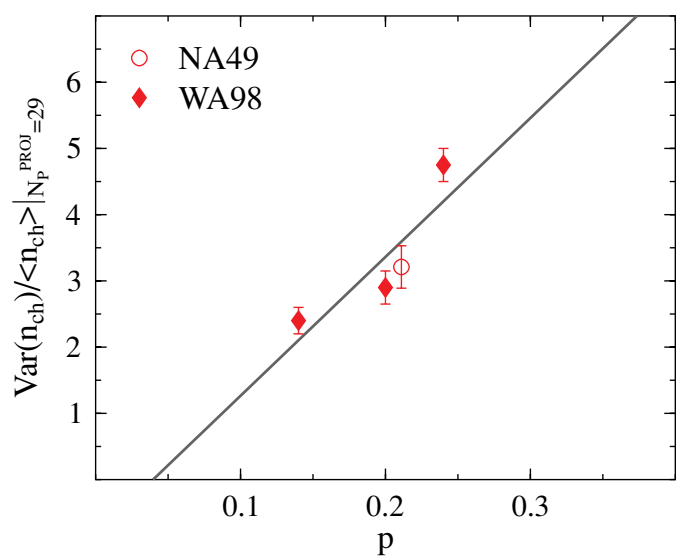

FIG. 15. (Color online) The scaled variance of the multiplicity distribution of all charged particles produced in semi-peripheral $\mathrm{Pb}+\mathrm{Pb}$ collisions as a function of the fraction of registered particles. The red diamonds correspond to the WA98 data [5]. The error bars represent the systematic uncertainties, the statistical errors are smaller than the symbols. The straight line shows a linear fit to the points. See text for the details.

NA49 acceptance filter. Figure 15 shows the scaled variance of the multiplicity distribution of all charged particles for $N_{P}^{\mathrm{PROJ}}=29$ from the NA49 and WA98 [5] experiments plotted versus the fraction $p$ of registered particles in the NA49 experiment and in the WA98 experiment [5]. The latter measured the multiplicity fluctuations of charged particles produced in $\mathrm{Pb}+\mathrm{Pb}$ collisions at $158 \mathrm{~A} \mathrm{GeV}$ in the central rapidity region. The predicted linear dependence of Eq. (15) is seen to agree with WA98 results [5]. The WA98 experiment used a centrality determination method that differs from that of NA49, i.e., the total transverse energy $E_{T}$ in the forward rapidity region was used to fix the centrality in the WA98 experiment. Nevertheless the results of NA49 and WA98 at the same value of $p$ agree (see Fig. 15).

The NA49 detector registers a fraction $p \approx 0.55$ of all produced particles. The fraction $p$ of registered particles can be decreased by cuts in the rapidity distribution. In the case of $p+p$ interactions, the scaled variance of the multiplicity distribution was calculated in the following rapidity intervals $\Delta y:(1.1,2.6),(0.85,3.8),(0.0,3.8),(-0.4,3.8)$, and $(-2.4,3.8)$, which correspond to fractions $p$ of registered particles equal to $0.18,0.25,0.41,0.48$, and 0.55 , respectively. Instead of cutting in rapidity one can change the fraction of registered particles by selecting a range in transverse momentum $p_{T}$ of the registered particles. The scaled variance of the multiplicity distribution was then calculated in the following $\Delta p_{T}(\mathrm{GeV})$ intervals: $(0.0,0.2),(0.0,0.4),(0.0$, $0.6)$ and in the full range corresponding to $p$ equal $0.2,0.37$, 0.41 , and 0.55 , respectively.

Figure 16 shows the scaled variance of the multiplicity distribution for negatively, positively, and all charged particles produced in $p+p$ collisions at $158 \mathrm{~A} \mathrm{GeV}$ as a function of the fraction $p$ of registered particles chosen by changing the rapidity and transverse-momentum intervals. One can clearly see the linear dependence described by Eq. (15). The dependence of the scaled variance on the fraction of registered

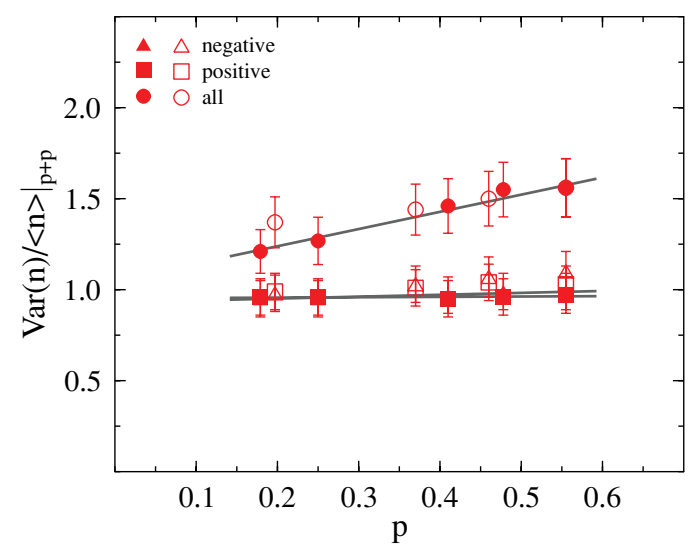

FIG. 16. (Color online) The scaled variance of the multiplicity distribution of negatively, positively, and all charged particles produced in $p+p$ collisions as a function of the fraction of registered particles chosen by change of rapidity (full symbols) or transversemomentum (open symbols) interval. The error bars represent the systematic uncertainties, the statistical errors are smaller than the symbols. The straight lines show the linear fits to the points.

particles is independent of the choice of rapidity or transverse momentum as cut variable.

The rise of the scaled variance of all charged particles with increasing acceptance can be presumably attributed to the effect of resonances that predominantly decay into oppositely charged particles.

Multiplicity distributions were measured in $p+p$ interactions in numerous experiments in a wide range of collision energies. An excellent compilation of $p+p$ data can be found in [36-38]. Figure 17 shows the scaled variance of multiplicity distributions of all charged particles in full phase space as a function of the collision energy. The extrapolated result from NA49 (Eq. (15)) is also shown in Fig. 17. The corrected scaled variance of all particles reaches a value of about 2 and fits well to the other data shown in this plot.

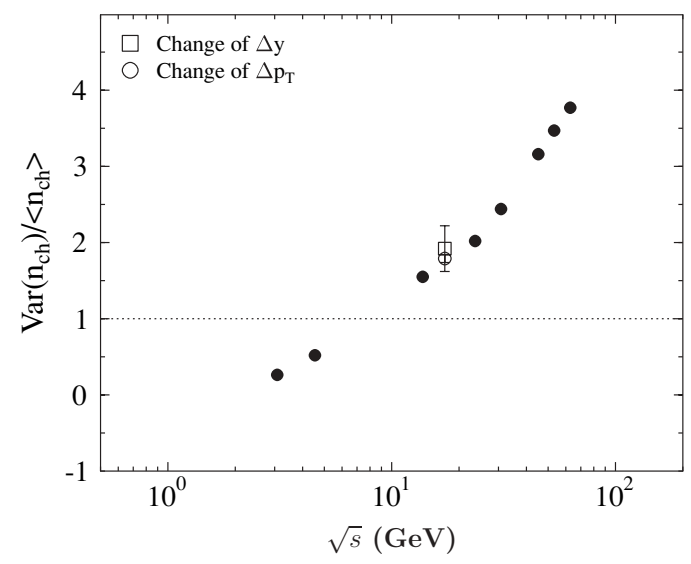

FIG. 17. The scaled variance of the multiplicity distribution of charged particles produced in $p+p$ interactions as a function of the center-of-mass energy. The full circles show a compilation of $p+p$ results (cf. [36-38]). The open symbols show our results obtained by the extrapolation by changing the rapidity $\Delta y$ or transversemomentum $\Delta p_{T}$ intervals. 


\section{COMPARISON WITH MODELS AND DISCUSSION}

Before closing our considerations we discuss possible origins of the strong dependence of $\operatorname{Var}(n) /\langle n\rangle$ on the collision centrality. In this context, it should be stated that the strong centrality dependence of the scaled variance found in our experimental study was completely unexpected. We do not know of any theoretical considerations that would have predicted the effect. However, after release of our preliminary data $[3,28]$ several attempts to explain them have been formulated [20,35,39-41]. Below the attempts are briefly presented.

We start the discussion with a reminder that the multiplicity fluctuations presented in this study were observed for a fixed number of the projectile participants. Although the average numbers of participants from the projectile and from the target are approximately equal in the collisions of identical nuclei, the number of target participants fluctuates even though the number of projectile participants is kept fixed. We also note that the multiplicity fluctuations were measured in the forward hemisphere $\left(1.1<y_{\text {c.m. }}<2.6\right)$. Keeping these remarks in mind, we note that the large multiplicity fluctuations seen in the forward rapidity window are caused either by dynamical fluctuations of the particle production process, which are present even at fixed number of projectile participants, or the multiplicity fluctuations in the forward hemisphere result from the fluctuations of target participant number (the number is not experimentally fixed) which are transferred to the forward hemisphere due to an unknown collision mechanism. The mechanism has to strongly couple the forward and backward hemispheres.

For the purpose of the further discussion, it is useful to divide the models of nucleus-nucleus collisions into three categories as proposed in Ref. [20]. There are transparency models where the projectile (target) participants, which are excited in the course of interaction, mostly contribute to the particle production in the forward (backward) hemisphere. Furthermore, there are (rather unrealistic) reflection models when the projectile (target) participants mostly contribute to the backward (forward) hemisphere. Finally, there are mixing models in which the projectile and target participants contribute significantly to both the backward and forward hemispheres.

In the transparency models, the observed multiplicity fluctuations can be caused only by the already mentioned dynamical fluctuations of the production process. In the reflection and mixing models, the fluctuations of the target participant number contribute to the multiplicity fluctuations in the forward hemisphere in addition to possible dynamical fluctuation. The models, which are represented in Figs. 11, 12, and 13, that is HIJING [29], HSD [30], and UrQMD [31] all belong to the transparency class. The results of VENUS [32] are very similar to those of HIJING, HSD, and UrQMD, even though the correlation between the forward and backward hemispheres is somewhat stronger in VENUS. The model calculations were performed with proper simulation of the VCAL response and the NA49 acceptance. The $E_{\text {Veto }}$ energy of a model event was calculated as the energy of the projectile spectators smeared by a Gaussian distribution with the width given by Eq. (10). The models, which produce approximately Poissonian multiplicity distributions independently of centrality, highly underestimate the observed multiplicity fluctuations in noncentral collisions. The models are unable to reproduce even qualitatively the centrality dependence of the scaled variance. This remark applies not only to the Monte Carlo models as HIJING [29], HSD [30], and UrQMD [31], which are based on string excitation and decay, but to any transparency model that does not assume correlations among secondary particles. For example, within a statistical model with fixed volume, where the electric charge is strictly conserved, the scaled variance of positive or negative particles varies in the range 0.5-1.0 [34]. The actual value depends on the thermal system's volume and the acceptance in which the multiplicity fluctuations are observed.

As already noted, the transparency models can produce large multiplicity fluctuations, if there are built in correlations among produced particles. The problem can be formulated in a quite general way.

The average multiplicity of secondaries is roughly proportional to the number of nucleons participating in the collision $N_{P}$ (cf. Fig. 5). Assuming that $N_{P}$ is proportional to the system's volume $V$, we have $\langle N\rangle=\bar{\rho} V$ with $\bar{\rho}$ being the constant density of produced particles. Defining the correlation function $v\left(\mathbf{r}_{1}-\mathbf{r}_{2}\right)=v(\mathbf{r})$ through the equation $\rho_{2}\left(\mathbf{r}_{1}, \mathbf{r}_{2}\right)=\rho\left(\mathbf{r}_{1}\right) \rho\left(\mathbf{r}_{2}\right)\left[1+v\left(\mathbf{r}_{1}-\mathbf{r}_{2}\right)\right]$, where $\rho_{2}\left(\mathbf{r}_{1}, \mathbf{r}_{2}\right)$ is the two-particle density, we get the desired formula

$$
\frac{\operatorname{Var}(N)}{\langle N\rangle}=1+\bar{\rho} \int_{V} d^{3} r v(\mathbf{r}),
$$

which tells us that the multiplicity fluctuations are related to the interparticle correlations. The multiplicity distribution is Poissonian, if the particles are emitted independently from each other $(v(\mathbf{r})=0)$.

It is not difficult to invent a correlation function $v(\mathbf{r})$ that, substituted in Eq. (16), reproduces the data shown in Fig. 11. Some functions are discussed in Ref. [39]. The correlation function has to be positive at small distances (attractive interaction) and negative at larger distances (repulsive interaction). For $\mathrm{Pb}+\mathrm{Pb}$ collisions the sign of the correlation changes at $r \approx 4 \mathrm{fm}$, which corresponds to $N_{P} \approx 70$ when $\operatorname{Var}(N) /\langle N\rangle$ reaches its maximum. For $r \gtrsim(300)^{1 / 3} \mathrm{fm} \approx$ $7 \mathrm{fm}$ the correlation function vanishes. Possible mechanisms responsible for such a correlation function were discussed in Ref. [39]. They include a combination of attractive and repulsive interaction (the prediction of this model is shown in Fig. 18(a)), percolation, dipole-dipole interaction, and nonextensive thermodynamics.

Specific percolation models, which produce strong correlations among secondaries and thus provide large multiplicity fluctuations, were discussed in Refs. [40,41]. The color strings, which are stretched in the course of the interaction, form multistring clusters. In $p+p$ collisions there are single-string clusters but in central $\mathrm{Pb}+\mathrm{Pb}$ collisions the density of strings is so high that the strings overlap and form one big cluster. The decrease of the scaled variance as a function of centrality is in this picture associated with the percolation phase transition, i.e., the appearance of the large cluster. The prediction of 


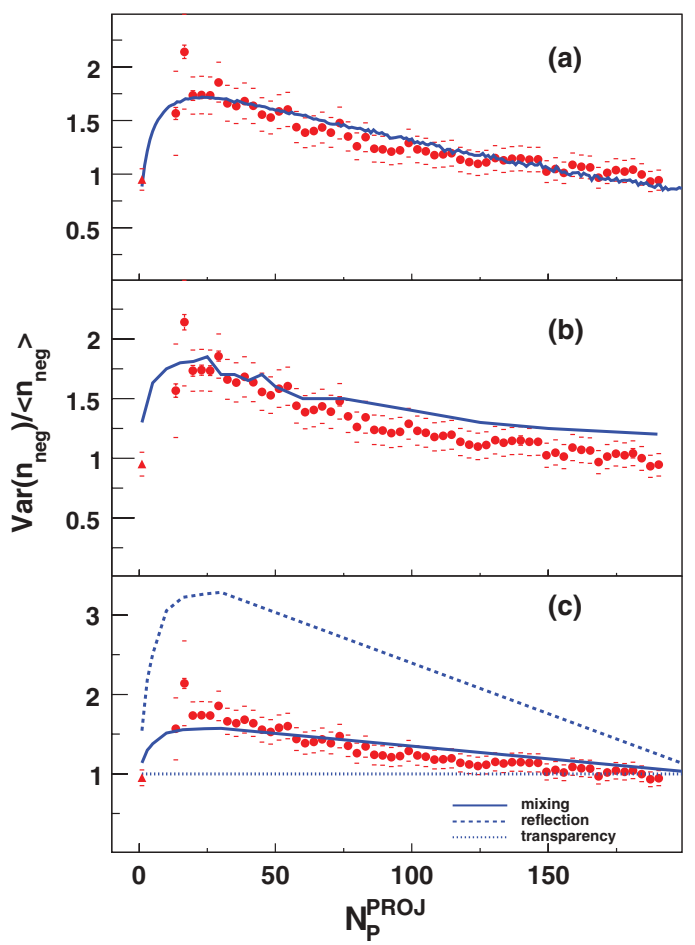

FIG. 18. (Color online) The scaled variance of the multiplicity distribution of negatively charged particles produced in $\mathrm{Pb}+\mathrm{Pb}$ minimum bias collisions as a function of the number of projectile participants. The data are compared to the predictions of the following models: (a) the model which assumes the inter-particle correlations caused by the combination of strong and electromagnetic interactions [39], (b) the percolation model [40], (c) the transparency, mixing and reflection models [20], see text for the details.

the model [40] is shown in Fig. 18(b) and reproduces the qualitative behavior of our experimental results.

Although the transparency models require strong interparticle correlations to comply with our data, the mixing and reflection models can produce large multiplicity fluctuations in the forward hemisphere due to the fluctuations of target participant number that is not fixed in our measurement. In Fig. 18(c) we show the prediction of the reflection and mixing models [35]. In the reflection model the target participants contribute to the forward hemisphere, and thus the fluctuations of the target participant number produce the multiplicity fluctuations of secondaries. As seen in Fig. 18(c), the reflection model strongly overestimates the observed scaled variance but the mixing models, where the target as well as projectile participants equally contribute to the forward and backward hemispheres, approximately agree with the data. The question arises whether the mixing models properly represent the dynamics of nucleus-nucleus collisions at high energies. A recent theoretical analysis of $d+\mathrm{Au}$ collisions at RHIC [42] suggests that the contribution of projectile participants indeed extends to the backward hemisphere, whereas that of target participants extends to the forward hemisphere.

In principle, the discussed models can predict multiplicity fluctuations not only in $\mathrm{Pb}+\mathrm{Pb}$ collisions but also in $p+p$,
$\mathrm{C}+\mathrm{C}$, and $\mathrm{Si}+\mathrm{Si}$ reactions. It remains to be seen whether they can provide a consistent description of the fluctuations in all reactions.

Finally we note that the transverse-momentum fluctuations measured in nuclear collisions at $158 \mathrm{~A} \mathrm{GeV} \mathrm{[10]} \mathrm{and}$ quantified by the $\Phi\left(p_{T}\right)$ show a similar centrality dependence with a maximum located close to that of the scaled variance of the multiplicity distribution. This behavior of the transverse-momentum fluctuations as a function of collision centrality was related in a superposition model [43] to the centrality dependence of the multiplicity fluctuations using the correlation of average $p_{T}$ and multiplicity observed in $p+p$ collisions [10]. Moreover, we note that the percolation string model also describes qualitatively the centrality dependence of $\Phi\left(p_{T}\right)$ [44].

\section{SUMMARY AND CONCLUSIONS}

Multiplicity fluctuations in the forward hemisphere were analyzed by studying the scaled variance of the multiplicity distributions in $p+p, \mathrm{C}+\mathrm{C}, \mathrm{Si}+\mathrm{Si}$, and $\mathrm{Pb}+\mathrm{Pb}$ collisions. $\mathrm{A}$ strong centrality dependence is seen for negatively, positively, and all charged particles. The number of projectile participants was used to determine the collision centrality. The scaled variance is close to unity for $p+p, \mathrm{C}+\mathrm{C}, \mathrm{Si}+\mathrm{Si}$, and central $\mathrm{Pb}+\mathrm{Pb}$ collisions. However, it increases significantly toward peripheral $\mathrm{Pb}+\mathrm{Pb}$ collisions. The magnitude of the scaled variance is similar for positively and negatively charged particles and is about 1.5 times larger for all charged particles.

The string-hadronic models of nuclear collisions (with or without color exchange and without string fusion) predict no dependence of the scaled variance on the number of projectile participants, and thus the models qualitatively disagree with the data. A maximum of the scaled variance appears for semiperipheral collisions when the forward and backward hemispheres are strongly coupled and fluctuations of the number of target participants contribute to the forward rapidity window. The observed scaled variance can also be reproduced within the models that assume strong dynamic correlations as, for example, in percolation phase transitions.

\section{ACKNOWLEDGMENTS}

This work was supported by the U.S. Department of Energy grant DE-FG03-97ER41020/A000, the Bundesministerium fur Bildung und Forschung, Germany (06F140), the Virtual Institute VI-146 of Helmholtz Gemeinschaft, Germany, the Polish State Committee for Scientific Research (1 P03B 097 29, 1 P03B 121 29, 1 P03B 006 30, 1 P03B 127 30), the Hungarian Scientific Research Foundation (T032648, T032293, T043514), the Hungarian National Science Foundation, OTKA (F034707), the Polish-German Foundation, the Korea Science \& Engineering Foundation (R01-2005000-10334-0), and the Bulgarian National Science Fund (Ph09/05). 
[1] J. C. Collins and M. J. Perry, Phys. Rev. Lett. 34, 1353 (1975).

[2] E. V. Shuryak, Phys. Rep. 61, 71 (1980).

[3] M. Gaździcki et al. (NA49 Collaboration), J. Phys. G 30, S701 (2004).

[4] H. Heiselberg, Phys. Rep. 351, 161 (2001).

[5] M. M. Aggarwal et al. (WA98 Collaboration), Phys. Rev. C 65, 054912 (2002).

[6] S. V. Afanasev et al. (NA49 Collaboration), Phys. Rev. Lett. 86, 1965 (2001).

[7] C. Roland et al. (NA49 Collaboration), J. Phys. G 30, S1381 (2004).

[8] J. Adams et al. (STAR Collaboration), Phys. Rev. C 68, 044905 (2003).

[9] Z. Chai et al. (PHOBOS Collaboration), J. Phys. Conf. Ser. 27, 128 (2005).

[10] T. Anticic et al. (NA49 Collaboration), Phys. Rev. C 70, 034902 (2004).

[11] D. Adamova et al. (CERES Collaboration), Nucl. Phys. A727, 97 (2003).

[12] J. Adams et al. (STAR Collaboration), Phys. Rev. C 71, 064906 (2005).

[13] S. S. Adler et al. (PHENIX Collaboration), Phys. Rev. Lett. 93, 092301 (2004).

[14] L. Stodolsky, Phys. Rev. Lett. 75, 1044 (1995).

[15] M. Stephanov, K. Rajagopal, and E. Shuryak, Phys. Rev. D 60, 114028 (1999).

[16] St. Mrówczyński, Phys. Lett. B430, 9 (1998).

[17] M. Stephanov, K. Rajagopal, and E. Shuryak, Phys. Rev. Lett. 81, 4816 (1998).

[18] M. Gaździcki, M. I. Gorenstein, and St. Mrówczyński, Phys. Lett. B585, 115 (2004).

[19] A. Białas, M. Błeszyński, and W. Czyż, Nucl. Phys. B111, 461 (1976).

[20] M. Gaździcki and M. Gorenstein, Phys. Lett. B640, 155 (2006).

[21] S. Afanasiev et al. (NA49 Collaboration), Nucl. Instrum. Methods A 430, 210 (1999).

[22] L. S. Barnby et al. (NA49 Collaboration), J. Phys. G 25, 469 (1999).

[23] C. Alt et al. (NA49 Collaboration), Eur. Phys. J. C 45, 343 (2006).

[24] S. V. Afanasiev et al. (The NA49 Collaboration), Phys. Rev. C 66, 054902 (2002).
[25] The acceptance of the VCAL at $158 \mathrm{~A} \mathrm{GeV}$ is approximately $\Theta<0.1$ deg for neutral particles (e.g., neutrons and photons) and due to the magnetic field $p>140 \mathrm{GeV} / c, p_{T}<0.4 \mathrm{GeV} / c$ for protons.

[26] M. Rybczyński, Ph.D. Dissertation, Swiętokrzyska Academy, Kielce, 2005.

[27] The response of the VCAL is different for different positions of the spectator's hits. It is caused by the construction of the calorimeter. The four photomultipliers, which gain the signal, are situated outside of the active area of the calorimeter sectors. Thus, the amplitude of the signal depends on the horizontal position, where the signal was generated, because of the absorption of the ultraviolet light in the scintillator.

[28] M. Rybczyński et al. (NA49 Collaboration), J. Phys. Conf. Ser. 5, 74 (2005).

[29] M. Gyulassy and X. N. Wang, Comput. Phys. Commun. 83, 307 (1994).

[30] W. Cassing and E. L. Bratkovskaya, Phys. Rep. 308, 65 (1999).

[31] M. Bleicher et al., J. Phys. G 25, 1859 (1999).

[32] K. Werner, Phys. Rep. 232, 87 (1993).

[33] M. Gaździcki, R. Szwed, G. Wrochna, and A. K. Wróblewski, Mod. Phys. Lett. A 6, 981 (1991).

[34] V. V. Begun, M. Gazdzicki, M. I. Gorenstein, and O. S. Zozulya, Phys. Rev. C 70, 034901 (2004).

[35] V. P. Konchakovski, S. Haussler, M. I. Gorenstein, E. L. Bratkovskaya, M. Bleicher, and H. Stocker, Phys. Rev. C 73, 034902 (2006).

[36] W. Thome et al. (Aachen-CERN-Heidelberg-Munich Collaboration), Nucl. Phys. B129, 365 (1977).

[37] J. Whitmore, Phys. Rep. 10, 273 (1974).

[38] J. Whitmore, Phys. Rep. 27, 187 (1976).

[39] M. Rybczyński and Z. Włodarczyk, J. Phys. Conf. Ser. 5, 238 (2005).

[40] L. Cunqueiro, E. G. Ferreiro, F. del Moral, and C. Pajares, Phys. Rev. C 72, 024907 (2005).

[41] P. Brogueira and J. Dias de Deus, Phys. Rev. C 72, 044903 (2005).

[42] A. Białas and W. Czyż, Acta Phys. Pol. B 36, 905 (2005).

[43] St. Mrówczyński, M. Rybczyński, and Z. Włodarczyk, Phys. Rev. C 70, 054906 (2004).

[44] E. G. Ferreiro, F. del Moral, and C. Pajares, Phys. Rev. C 69, 034901 (2004) 